\title{
Some identities on the weighted q-Euler numbers and $q$-Bernstein polynomials
}

\author{
Taekyun Kim", Young-Hee Kim ${ }^{1}$ and Cheon S Ryoo ${ }^{2}$
}

\author{
* Correspondence: tkkim@kw.ac.kr \\ ${ }^{1}$ Division of General Education- \\ Mathematics, Kwangwoon \\ University, Seoul 139-701, Korea \\ Full list of author information is \\ available at the end of the article
}

\begin{abstract}
Recently, Ryoo introduced the weighted q-Euler numbers and polynomials which are a slightly different Kim's weighted q-Euler numbers and polynomials(see C. S. Ryoo, A note on the weighted q-Euler numbers and polynomials, 2011]). In this paper, we give some interesting new identities on the weighted $q$-Euler numbers related to the q-Bernstein polynomials

2000 Mathematics Subject Classification - 11B68, 11S40, 11580
\end{abstract}

Keywords: Euler numbers and polynomials, $q$-Euler numbers and polynomials, weighted, $q$-Euler numbers and polynomials, Bernstein polynomials, $q$-Bernstein polynomials

\section{Introduction}

Let $p$ be a fixed odd prime number. Throughout this paper $\mathbb{Z}_{p}, \mathbb{Q}_{p}, \mathbb{C}$ and $\mathbb{C}_{p}$ will denote the ring of $p$-adic integers, the field of $p$-adic rational numbers, the complex number fields and the completion of algebraic closure of $\mathbb{Q}_{p}$, respectively. Let $\mathbb{N}$ be the set of natural numbers and $\mathbb{Z}_{+}=\mathbb{N} \cup\{0\}$. Let $v_{p}$ be the normalized exponential valuation of $\mathbb{C}_{p}$ with $|p|_{p}=p^{-v_{p}(p)}=\frac{1}{p}$. When one talks of $q$-extension, $q$ is variously considered as an indeterminate, a complex number $q \in \mathbb{C}$, or a $p$-adic number $q \in \mathbb{C}_{p}$. If $q \in$ $\mathbb{C}$, then one normally assumes $|q|<1$, and if $q \in \mathbb{C}_{p}$, then one normally assumes $|q-1|$ $p<1$. In this paper, the $q$-number is defined by

$$
[x]_{q}=\frac{1-q^{x}}{1-q}
$$

(see [1-19])

Note that $\lim _{q \rightarrow 1}[x]_{q}=x$ (see [1-19]). Let $f$ be a continuous function on $\mathbb{Z}_{p}$. For $\alpha \in$ $\mathbb{N}$ and $k, n \in \mathbb{Z}_{+}$, the weighted $p$-adic $q$-Bernstein operator of order $n$ for $f$ is defined by Kim as follows:

$$
\begin{aligned}
\mathbb{B}_{n, q}^{(\alpha)}(f \mid x) & =\sum_{k=0}^{n}\left(\begin{array}{l}
n \\
k
\end{array}\right) f\left(\frac{k}{n}\right)[x]_{q^{\alpha}}^{k}[1-x]_{q^{-\alpha}}^{n-k} \\
& =\sum_{k=0}^{n} f\left(\frac{k}{n}\right) B_{k, n}^{(\alpha)}(x, q), .
\end{aligned}
$$

\section{Springer}

(C) 2011 Kim et al; licensee Springer. This is an Open Access article distributed under the terms of the Creative Commons Attribution License (http://creativecommons.org/licenses/by/2.0), which permits unrestricted use, distribution, and reproduction in any medium, provided the original work is properly cited. 
Here $B_{k, n}^{(\alpha)}(x, q)=\left(\begin{array}{l}n \\ k\end{array}\right)[x]_{q^{\alpha}}^{k}[1-x]_{q^{-\alpha}}^{n-k}$ are called the $q$-Bernstein polynomials of degree $n$ with weighted $\alpha$.

Let $C\left(\mathbb{Z}_{p}\right)$ be the space of continuous functions on $\mathbb{Z}_{p}$. For $f \in C\left(\mathbb{Z}_{p}\right)$, the fermionic $q$ integral on $\mathbb{Z}_{p}$ is defined by

$$
I_{q}(f)=\int_{\mathbb{Z}_{p}} f(x) d \mu_{-q}(x)=\lim _{N \rightarrow \infty} \frac{1+q}{1+q^{p^{N}}} \sum_{x=0}^{p^{N}-1} f(x)(-q)^{x},
$$

see [5-19].

For $n \in \mathbb{N}$, by (2), we get

$$
q^{n} \int_{\mathbb{Z}_{p}} f(x+n) d \mu_{-q}(x)=(-1)^{n} \int_{\mathbb{Z}_{p}} f(x) d \mu_{-q}(x)+[2]_{q} \sum_{l=0}^{n-1}(-1)^{n-1-l} q^{l} f(l),
$$

see $[6,7]$.

Recently, by (2) and (3), Ryoo considered the weighted $q$-Euler polynomials which are a slightly different Kim's weighted $q$-Euler polynomials as follows:

$$
\int_{\mathbb{Z}_{p}}[x+\gamma]_{q^{\alpha}}^{n} d \mu_{-q}(y)=E_{n, q}^{(\alpha)}(x), \text { for } n \in \mathbb{Z}_{+} \text {and } \alpha \in \mathbb{Z},
$$

see [17].

In the special case, $x=0, E_{n, q}^{(\alpha)}(0)=E_{n, q}^{(\alpha)}$ are called the $n$-th $q$-Euler numbers with weight $\alpha$ (see [14]).

From (4), we note that

$$
E_{n, q}^{(\alpha)}(x)=\frac{[2]_{q}}{\left(1-q^{\alpha}\right)^{n}} \sum_{l=0}^{n}\left(\begin{array}{l}
n \\
l
\end{array}\right)(-1)^{l} \frac{q^{\alpha l x}}{1+q^{\alpha l+1}},
$$

see [17].

and

$$
E_{n, q}^{(\alpha)}(x)=\sum_{l=0}^{n}\left(\begin{array}{l}
n \\
l
\end{array}\right)[x]_{q^{\alpha}}^{n-l} q^{\alpha l x} E_{l, q}^{(\alpha)}
$$

see [17].

That is, (6) can be written as

$$
E_{n, q}^{(\alpha)}(x)=\left(q^{\alpha x} E_{q}^{(\alpha)}+[x]_{q^{\alpha}}\right)^{n}, n \in \mathbb{Z}_{+} .
$$

with usual convention about replacing $\left(E_{q}^{(\alpha)}\right)^{n}$ by $E_{n, q^{*}}^{(\alpha)}$

In this paper we study the weighted $q$-Bernstein polynomials to express the fermionic $q$-integral on $\mathbb{Z}_{p}$ and investigate some new identities on the weighted $q$-Euler numbers related to the weighted $q$-Bernstein polynomials.

\section{2. q-Euler numbers with weight $\alpha$}

In this section we assume that $\alpha \in \mathbb{N}$ and $q \in \mathbb{C}$ with $|q|<1$.

Let $F_{q}(t, x)$ be the generating function of $q$-Euler polynomials with weight $\alpha$ as followings: 


$$
F_{q}(t, x)=\sum_{n=0}^{\infty} E_{n, q}^{(\alpha)}(x) \frac{t^{n}}{n !}
$$

By (5) and (8), we get

$$
\begin{aligned}
F_{q}(t, x) & =\sum_{n=0}^{\infty}\left(\frac{[2]_{q}}{\left(1-q^{\alpha}\right)^{n}} \sum_{l=0}^{n}\left(\begin{array}{c}
n \\
l
\end{array}\right)(-1)^{l} \frac{q^{\alpha l x}}{1+q^{\alpha l+1}}\right) \frac{t^{n}}{n !} \\
& =[2]_{q} \sum_{m=0}^{\infty}(-1)^{m} q^{m} e^{[x+m]_{q} \alpha t} .
\end{aligned}
$$

In the special case, $x=0$, let $F_{q}(t, 0)=F_{q}(t)$. Then we obtain the following difference equation.

$$
q F_{q}(t, 1)+F_{q}(t)=[2]_{q} .
$$

Therefore, by (8) and (10), we obtain the following proposition.

Proposition 1. For $n \in \mathbb{Z}_{+}$, we have

$$
E_{0, q}^{(\alpha)}=1, \text { and } q E_{n, q}^{(\alpha)}(1)+E_{n, q}^{(\alpha)}=0 \text { if } n>0 .
$$

By (6), we easily get the following corollary.

Corollary 2. For $n \in \mathbb{Z}_{+}$, we have

$$
E_{0, q}^{(\alpha)}=1, \quad \text { and } q\left(q^{\alpha} E_{q}^{(\alpha)}+1\right)^{n}+E_{n, q}^{(\alpha)}=0 \text { if } n>0,
$$

with usual convention about replacing $\left(E_{q}^{(\alpha)}\right)^{n}$ by $E_{n, q^{\circ}}^{(\alpha)}$.

From (9), we note that

$$
F_{q^{-1}}(t, 1-x)=F_{q}\left(-q^{\alpha} t, x\right) .
$$

Therefore, by (11), we obtain the following lemma.

Lemma 3. Let $n \in \mathbb{Z}_{+}$. Then we have

$$
E_{n, q^{-1}}^{(\alpha)}(1-x)=(-1)^{n} q^{\alpha n} E_{n, q}^{(\alpha)}(x) .
$$

By Corollary 2, we get

$$
\begin{aligned}
q^{2} E_{n, q}^{(\alpha)}(2)-q^{2}-q & =q^{2} \sum_{l=0}^{n}\left(\begin{array}{c}
n \\
l
\end{array}\right) q^{\alpha l}\left(q^{\alpha} E_{q}^{(\alpha)}+1\right)^{l}-q^{2}-q \\
& =-q \sum_{l=1}^{n}\left(\begin{array}{c}
n \\
l
\end{array}\right) q^{\alpha l} E_{l, q}^{(\alpha)}-q \\
& =-q \sum_{l=0}^{n}\left(\begin{array}{c}
n \\
l
\end{array}\right) q^{\alpha l} E_{l, q}^{(\alpha)} \\
& =-q E_{n, q}^{(\alpha)}(1)=E_{n, q}^{(\alpha)} \text { if } n>0 .
\end{aligned}
$$

Therefore, by (12), we obtain the following theorem.

Theorem 4. For $n \in \mathbb{N}$, we have

$$
E_{n, q}^{(\alpha)}(2)=\frac{1}{q^{2}} E_{n, q}^{(\alpha)}+\frac{1}{q}+1 .
$$


Theorem 4 is important to study the relations between $q$-Bernstein polynomials and the weighted $q$-Euler number in the next section.

\section{Weighted q-Euler numbers concerning $\boldsymbol{q}$-Bernstein polynomials}

In this section we assume that $\alpha \in \mathbb{Z}_{p}$ and $q \in \mathbb{C}_{p}$ with $|1-q|_{p}<1$.

From (2), (3) and (4), we note that

$$
\begin{aligned}
q \int_{\mathbb{Z}_{p}}[1-x]_{q^{-\alpha}}^{n} d \mu_{-q}(x) & =(-1)^{n} q^{\alpha n+1} \int_{\mathbb{Z}_{p}}[x-1]_{q^{\alpha}}^{n} d \mu_{-q}(x) \\
& =q \sum_{l=0}^{n}\left(\begin{array}{c}
n \\
l
\end{array}\right)(-1)^{l} \int_{\mathbb{Z}_{p}}[x]_{q^{\alpha}}^{l} d \mu_{-q}(x) .
\end{aligned}
$$

Therefore, by (13) and Lemma 3, we obtain the following theorem.

Theorem 5. For $n \in \mathbb{Z}_{+}$, we get

$$
\begin{aligned}
q \int_{\mathbb{Z}_{p}}[1-x]_{q^{-\alpha}}^{n} d \mu_{-q}(x) & =(-1)^{n} q^{\alpha n+1} E_{n, q}^{(\alpha)}(-1)=q E_{n, q^{-1}}^{(\alpha)} \\
& =q \sum_{l=0}^{n}\left(\begin{array}{c}
n \\
l
\end{array}\right)(-1)^{l} E_{l, q}^{(\alpha)} .
\end{aligned}
$$

Let $n \in \mathbb{N}$. Then, by Theorem 4 , we obtain the following corollary.

Corollary 6. For $n \in \mathbb{N}$, we have

$$
\begin{aligned}
\int_{\mathbb{Z}_{p}}[1-x]_{q^{-\alpha}}^{n} d \mu_{-q}(x) & =E_{n, q^{-1}}^{(\alpha)}(2) \\
& =q^{2} E_{n, q^{-1}}^{(\alpha)}+[2]_{q} .
\end{aligned}
$$

For $x \in \mathbb{Z}_{p}$, the $p$-adic $q$-Bernstein polynomials with weight $\alpha$ of degree $n$ are given by

$$
B_{k, n}^{(\alpha)}(x, q)=\left(\begin{array}{l}
n \\
k
\end{array}\right)[x]_{q^{\alpha}}^{k}[1-x]_{q^{-\alpha}}^{n-k}, \text { where } n, k \in \mathbb{Z}_{+}
$$

see [9].

From (14), we can easily derive the following symmetric property for $q$-Bernstein polynomials:

$$
B_{k, n}^{(\alpha)}(x, q)=B_{n-k, n}^{(\alpha)}\left(1-x, q^{-1}\right),
$$

see $[11]$

By (15), we get

$$
\begin{aligned}
\int_{\mathbb{Z}_{p}} B_{k, n}^{(\alpha)}(x, q) d \mu_{-q}(x) & =\int_{\mathbb{Z}_{p}} B_{n-k, n}^{(\alpha)}\left(1-x, q^{-1}\right) d \mu_{-q}(x) \\
& =\left(\begin{array}{l}
n \\
k
\end{array}\right) \sum_{l=0}^{k}\left(\begin{array}{l}
k \\
l
\end{array}\right)(-1)^{k+l} \int_{\mathbb{Z}_{p}}[1-x]_{q^{-\alpha}}^{n-l} d \mu_{-q}(x) .
\end{aligned}
$$


Let $n, k \in \mathbb{Z}_{+}$with $n>k$. Then, by (16) and Corollary 6, we have

$$
\begin{aligned}
& \int_{\mathbb{Z}_{p}} B_{k, n}^{(\alpha)}(x, q) d \mu_{-q}(x) \\
& =\left(\begin{array}{l}
n \\
k
\end{array}\right) \sum_{l=0}^{k}\left(\begin{array}{l}
k \\
l
\end{array}\right)(-1)^{k+l}\left(q^{2} E_{n-l, q^{-1}}^{(\alpha)}+[2]_{q}\right) \\
& = \begin{cases}q^{2} E_{n, q^{-1}}^{(\alpha)}+[2]_{q^{\prime}} & \text { if } k=0, \\
q^{2}\left(\begin{array}{l}
n \\
k
\end{array}\right) \sum_{l=0}^{k}\left(\begin{array}{l}
k \\
l
\end{array}\right)(-1)^{k+l} E_{n-l, q^{-1}}^{(\alpha)}, & \text { if } k>0 .\end{cases}
\end{aligned}
$$

Taking the fermionic $q$-integral on $\mathbb{Z}_{p}$ for one weighted $q$-Bernstein polynomials in (14), we have

$$
\begin{aligned}
\int_{\mathbb{Z}_{p}} B_{k, n}^{(\alpha)}(x, q) d \mu_{-q}(x) & =\left(\begin{array}{l}
n \\
k
\end{array}\right) \int_{\mathbb{Z}_{p}}[x]_{q^{\alpha}}^{k}[1-x]_{q^{-\alpha}}^{n-k} d \mu_{-q}(x) \\
& =\left(\begin{array}{l}
n \\
k
\end{array}\right) \sum_{l=0}^{n-k}\left(\begin{array}{c}
n-k \\
l
\end{array}\right)(-1)^{l} \int_{\mathbb{Z}_{p}}[x]_{q^{\alpha}}^{k+l} d \mu_{-q}(x) \\
& =\left(\begin{array}{l}
n \\
k
\end{array}\right) \sum_{l=0}^{n-k}\left(\begin{array}{c}
n-k \\
l
\end{array}\right)(-1)^{l} E_{l+k, q}^{(\alpha)} .
\end{aligned}
$$

Therefore, by comparing the coefficients on the both sides of (17) and (18), we obtain the following theorem.

Theorem 7. For $n, k \in \mathbb{Z}_{+}$with $n>k$, we have

$$
\sum_{l=0}^{n-k}(-1)^{l}\left(\begin{array}{c}
n-k \\
l
\end{array}\right) E_{l+k, q}^{(\alpha)}= \begin{cases}q^{2} E_{n, q^{-1}}^{(\alpha)}+[2]_{q^{\prime}} & \text { if } k=0 \\
q^{2} \sum_{l=0}^{k}\left(\begin{array}{c}
k \\
l
\end{array}\right)(-1)^{k+l} E_{n-l, q^{-1}}^{(\alpha)}, & \text { if } k>0 .\end{cases}
$$

Let $n_{1}, n_{2}, k \in \mathbb{Z}_{+}$with $n_{1}+n_{2}>2 k$. Then we see that

$$
\begin{aligned}
& \int_{\mathbb{Z}_{p}} B_{k, n_{1}}^{(\alpha)}(x, q) B_{k, n_{2}}^{(\alpha)}(x, q) d \mu_{-q}(x) \\
& =\left(\begin{array}{c}
n_{1} \\
k
\end{array}\right)\left(\begin{array}{c}
n_{2} \\
k
\end{array}\right) \sum_{l=0}^{2 k}\left(\begin{array}{c}
2 k \\
l
\end{array}\right)(-1)^{l+2 k} \int_{\mathbb{Z}_{p}}[1-x]_{q^{-\alpha}}^{n_{1}+n_{2}-l} d \mu_{-q}(x) \\
& =\left(\begin{array}{c}
n_{1} \\
k
\end{array}\right)\left(\begin{array}{c}
n_{2} \\
k
\end{array}\right) \sum_{l=0}^{2 k}\left(\begin{array}{c}
2 k \\
l
\end{array}\right)(-1)^{l+2 k}\left(q^{2} E_{n_{1}+n_{2}-l, q^{-1}}^{(\alpha)}+[2]_{q}\right) .
\end{aligned}
$$

By the binomial theorem and definition of $q$-Bernstein polynomials, we get

$$
\begin{aligned}
& \int_{\mathbb{Z}_{p}} B_{k, n_{1}}^{(\alpha)}(x, q) B_{k, n_{2}}^{(\alpha)}(x, q) d \mu_{-q}(x) \\
& =\left(\begin{array}{c}
n_{1} \\
k
\end{array}\right)\left(\begin{array}{c}
n_{2} \\
k
\end{array}\right) \sum_{l=0}^{n_{1}+n_{2}-2 k}(-1)^{l}\left(\begin{array}{c}
n_{1}+n_{2}-2 k \\
l
\end{array}\right) \int_{\mathbb{Z}_{p}}[x]_{q^{\alpha}}^{2 k+l} d \mu_{-q}(x) \\
& =\left(\begin{array}{c}
n_{1} \\
k
\end{array}\right)\left(\begin{array}{c}
n_{2} \\
k
\end{array}\right) \sum_{l=0}^{n_{1}+n_{2}-2 k}(-1)^{l}\left(\begin{array}{c}
n_{1}+n_{2}-2 k \\
l
\end{array}\right) E_{2 k+l, q}^{(\alpha)} .
\end{aligned}
$$


By comparing the coefficients on the both sides of (19) and (20), we obtain the following theorem.

Theorem 8. Let $n_{1}, n_{2}, k \in \mathbb{Z}_{+}$with $n_{1}+n_{2}>2 k$. Then we have

$$
\begin{aligned}
& \sum_{l=0}^{n_{1}+n_{2}-2 k}(-1)^{l}\left(\begin{array}{c}
n_{1}+n_{2}-2 k \\
l
\end{array}\right) E_{2 k+l, q}^{(\alpha)} \\
= & \begin{cases}q^{2} E_{n_{1}+n_{2}, q^{-1}}^{(\alpha)}+[2]_{q^{\prime}} & \text { if } k=0, \\
q^{2} \sum_{l=0}^{2 k}\left(\begin{array}{c}
2 k \\
l
\end{array}\right)(-1)^{2 k+l} E_{n_{1}+n_{2}-l, q^{-1}}^{(\alpha)}, & \text { if } k>0 .\end{cases}
\end{aligned}
$$

Let $s \in \mathbb{N}$ with $s \geq 2$. For $n_{1}, n_{2}, \ldots, n_{s}, k \in \mathbb{Z}_{+}$with $n_{1}+\ldots+n_{s}>s k$, we have

$$
\begin{aligned}
& \int_{\mathbb{Z}_{p}} \underbrace{B_{k, n_{1}}^{(\alpha)}(x, q) \cdots B_{k, n_{s}}^{(\alpha)}(x, q)}_{s-\text { times }} d \mu_{-q}(x) \\
& =\left(\begin{array}{c}
n_{1} \\
k
\end{array}\right) \cdots\left(\begin{array}{c}
n_{s} \\
k
\end{array}\right) \int_{\mathbb{Z}_{p}}[x]_{q^{\alpha}}^{s k}[1-x]_{q^{-\alpha}}^{n_{1}+\cdots+n_{s}-s k} d \mu_{-q}(x) \\
& =\left(\begin{array}{c}
n_{1} \\
k
\end{array}\right) \cdots\left(\begin{array}{c}
n_{s} \\
k
\end{array}\right) \sum_{l=0}^{s k}\left(\begin{array}{c}
s k \\
l
\end{array}\right)(-1)^{l+s k} \int_{\mathbb{Z}_{p}}[1-x]_{q^{-\alpha}}^{n_{1}+\cdots+n_{s}-l} d \mu_{-q}(x) \\
& =\left(\begin{array}{c}
n_{1} \\
k
\end{array}\right) \cdots\left(\begin{array}{c}
n_{s} \\
k
\end{array}\right) \sum_{l=0}^{s k}\left(\begin{array}{c}
s k \\
l
\end{array}\right)(-1)^{l+s k}\left(q^{2} E_{n_{1}+\cdots+n_{s}-l, q^{-1}}^{(\alpha)}+[2]_{q}\right) .
\end{aligned}
$$

From the binomial theorem and the definition of $q$-Bernstein polynomials, we note that

$$
\begin{aligned}
& \int_{\mathbb{Z}_{p}} \underbrace{B_{k, n_{1}}^{(\alpha)}(x, q) \cdots B_{k, n_{s}}^{(\alpha)}(x, q)}_{s-\text { times }} d \mu_{-q}(x) \\
& =\left(\begin{array}{c}
n_{1} \\
k
\end{array}\right) \cdots\left(\begin{array}{c}
n_{s} \\
k
\end{array}\right) \sum_{l=0}^{n_{1}+\cdots+n_{s}-s k}(-1)^{l}\left(\begin{array}{c}
n_{1}+\cdots+n_{s}-s k \\
l
\end{array}\right) \int_{\mathbb{Z}_{p}}[x]_{q^{\alpha}}^{s k+l} d \mu_{-q}(x) \\
& =\left(\begin{array}{c}
n_{1} \\
k
\end{array}\right) \cdots\left(\begin{array}{c}
n_{s} \\
k
\end{array}\right) \sum_{l=0}^{n_{1}+\cdots+n_{s}-s k}(-1)^{l}\left(\begin{array}{c}
n_{1}+\cdots+n_{s}-s k \\
l
\end{array}\right) E_{s k+l, q}^{(\alpha)} .
\end{aligned}
$$

Therefore, by (21) and (22), we obtain the following theorem.

Theorem 9. Let $s \in \mathbb{N}$ with $s \geq 2$. For $n_{1}, n_{2}, \ldots, n_{s}, k \in \mathbb{Z}_{+}$with $n_{1}+\ldots+n_{s}>s k$, we have

$$
\begin{aligned}
& \sum_{l=0}^{n_{1}+\cdots+n_{s}-s k}(-1)^{l}\left(\begin{array}{c}
n_{1}+\cdots+n_{s}-s k \\
l
\end{array}\right) E_{s k+l, q}^{(\alpha)} \\
= & \left\{\begin{array}{cc}
q^{2} E_{n_{1}+\cdots+n_{s}, q^{-1}}^{(\alpha)}+[2]_{q^{\prime}} & \text { if } k=0, \\
q^{2} \sum_{l=0}^{s k}\left(\begin{array}{c}
s k \\
l
\end{array}\right)(-1)^{l+s k} E_{n_{1}+\cdots+n_{s}-l, q^{-1}}^{(\alpha)}, & \text { if } k>0 .
\end{array}\right.
\end{aligned}
$$




\section{Author details}

'Division of General Education-Mathematics, Kwangwoon University, Seoul 139-701, Korea ${ }^{2}$ Department of Mathematics, Hannam University, Daejeon 306-791, Korea

\section{Authors' contributions}

All authors contributed equally to the manuscript and read and approved the finial manuscript.

\section{Competing interests}

The authors declare that they have no competing interests. Acknowledgment The authors would like to express their sincere gratitude to referee for his/her valuable comments.

Received: 18 February 2011 Accepted: 20 September 2011 Published: 20 September 2011

\section{References}

1. Bayad, A: Modular properties of elliptic Bernoulli and Euler functions. Adv Stud Contemp Math. 20, 389-401 (2010)

2. Cangul, IN, Kurt, V, Ozden, H, Simsek, Y: On the higher-order w-q-Genocchi numbers. Adv Stud Contemp Math. 19, 39-57 (2009)

3. Govil, NK, Gupta, V: Convergence of q-Meyer-Konig-Zeller-Durrmeyer operators. Adv Stud Contemp Math. 19, 97-108 (2009)

4. Jang, LC, Kim, W-J, Simsek, $Y$ : A study on the $p$-adic integral representation on $Z_{p}$ associated with Bernstein and Bernoulli polynomials, Advances in Difference Equations. 2010, 6 (2010) Article ID 163217

5. Jang, LC: A note on Norlund-type twisted $q$-Euler polynomials and numbers of higher order associated with fermionic invariant $q$-integrals. J Inequal Appl 2010, 12 (2010). Art. ID 417452

6. Kim, T: The modified q-Euler numbers and polynomials. Adv Stud Contemp Math. 16, 161-170 (2008)

7. Kim, T: Some identities on the $q$-Euler polynomials of higher order and $q$-Stirling numbers by the fermionic $p$-adic integral on Z . Russ J Math phys. 16, 484-491 (2009). doi:10.1134/S1061920809040037

8. Kim, T: Barnes type multiple $q$-zeta function and $q$-Euler polynomials. J phys A: Math Theor 43, 11 (2010). 255201

9. Kim, T: A note on q-Bernstein polynomials. Russ J Math phys. 18, 41-50 (2011)

10. Kim, T: q-Bernoulli numbers and polynomials associated with Gaussian binomial coefficients. Russ J Math Phys. 15, 51-57 (2008)

11. Kim, T, Lee, B, Choi, J, Kim, YH, Rim, SH: On the q-Euler numbers and weighted q-Bernstein polynomials. Adv Stud Contemp Math. 21, 13-18 (2011)

12. Kim, T: New approach to q- Euler polynomials of higher order. Russ J Math phys. 17, 218-225 (2010). doi:10.1134/ S1061920810020068

13. Kim, T, Lee, B, Choi, J, Kim, YH: A new approach of $q$-Euler numbers and polynomials. Proc Jangjeon Math Soc. 14, 7-14 (2011)

14. Kim, T, Choi, J, Kim, YH: q-Bernstein polynomials associated with $q$-Stirling numbers and Carlitz's $q$-Bernoulli numbers, Abstract and Applied Analysis. 2010, 11 (2010) Article ID 150975

15. Ozden, $H$, Simsek, Y: A new extension of $q$-Euler numbers and polynomials related to their interpolation functions. Appl Math Lett. 21, 934-939 (2008). doi:10.1016/j.aml.2007.10.005

16. Ryoo, CS: On the generalized Barnes type multiple $q$-Euler polynomials twisted by ramified roots of unity. Proc Jangjeon Math Soc. 13, 255-263 (2010)

17. Ryoo, CS: A note on the weighted $q$-Euler numbers and polynomials. Adv Stud Contemp Math. 21, 47-54 (2011)

18. Rim, S-H, Jin, J-H, Moon, E-J, Lee, S-J: On multiple interpolation function of the q-Genocchi polynomials. J Inequal Appl 13 (2010). Art ID 351419

19. Simsek, Y, Acikgoz, M: A new generating function of (q-) Bernstein-type polynomials and their interpolation function. Abstr Appl Anal 12 (2010). Art. ID 769095

doi:10.1186/1029-242X-2011-64

Cite this article as: Kim et al: Some identities on the weighted q-Euler numbers and q-Bernstein polynomials. Journal of Inequalities and Applications 2011 2011:64.

\section{Submit your manuscript to a SpringerOpen ${ }^{\odot}$ journal and benefit from:}

- Convenient online submission

- Rigorous peer review

- Immediate publication on acceptance

- Open access: articles freely available online

- High visibility within the field

- Retaining the copyright to your article

Submit your next manuscript at $\boldsymbol{s p r i n g e r o p e n . c o m ~}$ 